\title{
Interpersonal relationships modulate outcome evaluation in a social comparison context: The pain and pleasure of intimacy
}

\author{
Huoyin Zhang ${ }^{1,2} \cdot$ Mingming Zhang ${ }^{1} \cdot$ Jiachen $\mathrm{Lu}^{1,2} \cdot$ Lili Zhao $^{1} \cdot$ Dongfang Zhao ${ }^{1} \cdot$ Chuan Xiao $^{1} \cdot$ Ruolei Gu$^{3,4}$. \\ Wenbo Luo ${ }^{1}$
}

Published online: 10 December 2019

(C) The Psychonomic Society, Inc. 2019

\begin{abstract}
Previous research has revealed that interpersonal relationships and social comparisons play important roles in evaluating outcomes. To our knowledge, how interpersonal relationships influence the process of outcome evaluations in a social comparison context remains largely unclear. In the current study, participants engaged in a simple gambling task with an acquaintance or a stranger and received outcome feedback. Behavioral results showed that participants' satisfaction level was sensitive to the outcome of their fellow players when participants won. In this condition, the satisfaction level was greater when their fellow players won rather than lost. Moreover, the satisfaction level was greater when their friends won compared with when a stranger won. Event-related potential (ERP) results showed that when participants won, the feedback-related negativity (FRN) was more negative going for other's losses than for other's gains. Moreover, the FRN was also more negative going for a stranger's gains than a friend's gains. In contrast, in the self-loss condition, the FRN was more negative going for other's gains than for other's losses regardless of the type of interpersonal relationship. These FRN findings indicate that the experience of other's outcomes is sensitive to participants' own outcomes. Importantly, the interpersonal relationship only showed its influence when both the self and others received monetary gains. Finally, the P300 registered participants' attention resource allocation toward monetary gains for themselves and for others, which was unaffected by the interpersonal relationship. This work reveals that outcome evaluation in various social comparison contexts is sensitive to the difference in interpersonal relationship in its early stage, labeled by the FRN.
\end{abstract}

Keywords Interpersonal relationship - Outcome evaluation - Social comparison · Feedback-related negativity (FRN) · P300 · Gambling task

Social comparison is ubiquitous in everyday life, as human beings understand themselves by comparing their own personal

Electronic supplementary material The online version of this article (https://doi.org/10.3758/s13415-019-00756-6) contains supplementary material, which is available to authorized users.

Ruolei Gu

gurl@psych.ac.cn

Wenbo Luo

luowb@lnnu.edu.cn

1 Research Center of Brain and Cognitive Neuroscience, Liaoning Normal University, Dalian 116029, China

2 School of Psychology, Shenzhen University, Shenzhen 518061, China

3 CAS Key Laboratory of Behavioral Science, Institute of Psychology, Chinese Academy of Sciences, Beijing 100101, China

4 Department of Psychology, University of Chinese Academy of Sciences, Beijing 100101, China benefits, abilities, and opinions with others (Festinger, 1954). When evaluating personal profits, individuals might be concerned with the financial gains and losses of others in interpersonal interactions; people might not be satisfied with the rewards they receive when they are aware that others have received much more (Shen, Jin, \& Ma, 2013).

In daily life, we interact with various kinds of people, including those we know as well as with strangers (Schreuders, Klapwijk, Will, \& Guroglu, 2018). Would people still care about receiving as much benefits as others when comparing with familiar persons? Additionally, would comparing with significant others as opposed to comparing with strangers involve overlapping or distinct neural correlates? This study aims to explore the above questions from a neuroscientific perspective.

\section{Social comparison}

Previous studies have found that the psychological effect of relative income is stronger than the effect of absolute income 
(Ball \& Chernova, 2008; Dvash, Gilam, Ben-Ze'ev, Hendler, $\&$ Shamay-Tsoory, 2010), which suggests that the value of an outcome depends largely on its social context. Studies have shown that social comparison on social networking sites (e.g., Facebook and Instagram) can have negative psychological consequences. For example, the observation of other individuals' positively presented material may have detrimental consequences (Appel, Gerlach, \& Crusius, 2016; Hu, Kim, Siwek, \& Wilder, 2017a).

There has been a substantial amount of research on the neural underpinnings of social comparison with strangers. Functional magnetic resonance imaging (fMRI) studies have identified consistent involvement of the ventral striatum (VS) and ventromedial prefrontal cortex (vmPFC) in downward comparison (comparing to worse-off others). Moreover, consistent involvement of the anterior insula (AI) and dorsal anterior cingulate cortex (dACC) has been observed in upward comparison (comparing to better-off others). In this regard, downward comparison might signal positive norm prediction errors encoded in the VS and VmPFC and trigger pleasant feelings (e.g., schadenfreude). In contrast, upward comparison might signal negative norm prediction errors detected by the $\mathrm{AI}$ and dACC and evoke unpleasant feelings (e.g., envy; Fliessbach et al., 2007; Luo, Eickhoff, Hetu, \& Feng, 2018).

Also, much work has been conducted on the temporal processing of outcome evaluation in social comparison contexts. Event-related potential (ERP) studies have shown that the feedback-related negativity (FRN) and P300 component were sensitive to the level of difference in outcome between oneself and others (Luo et al., 2015; Wu, Zhang, Elieson, \& Zhou, 2012). The FRN is a negative-deflecting amplitude distributed in the frontocentral regions of the scalp, reaching its peak between 200 and $300 \mathrm{~ms}$ postonset of the feedback stimulus (Gehring \& Willoughby, 2002; Miltner, Braun, \& Coles, 1997). The amplitude of the FRN is more negative going for negative outcomes (e.g., monetary loss and incorrect feedback) rather than for positive outcomes (Gehring \& Willoughby, 2002; Hauser et al., 2014). Furthermore, this component is sensitive to the violation of expectancy (i.e., unexpected outcomes elicit a more negative-going FRN than expected ones (see Chen, Wu, Tong, Guan, \& Zhou, 2012; Oliveira, McDonald, \& Goodman, 2007). However, recent work has emphasized that the activity in the time range of the FRN may instead reflect an underlying positivity in response to rewards that is reduced or absent in response to losses (i.e., a reward-related positivity, also called RewP). The amplitude of the RewP is more positive going for positive outcomes (e.g., monetary gain and correct feedback) rather than negative outcomes (Foti, Weinberg, Dien, \& Hajcak, 2011b; Proudfit, 2015; Weinberg, Luhmann, Bress, \& Hajcak, 2012). Another component associated with outcome evaluation is the $\mathrm{P} 300$, a large positive component occurring in the 300-600-ms time window after stimulus onset, with its generators over parietal and occipital sites (Kutas, Mccarthy, \& Donchin, 1977; Sutton, Braren, Zubin, \& John, 1965). This component has been associated with various cognitive functions, particularly attentional allocation (Hajcak, Holroyd, Moser, \& Simons, 2005) and motivational/affective salience (Nieuwenhuis, Aston-Jones, \& Cohen, 2005). Arguably, these two functions may be inherently related, such that motivationally significant stimuli preferentially engage attention (Engelmann \& Pessoa, 2007; Gu, Zhang, Luo, Wang, \& Broster, 2018).

Previous ERP studies have explored social comparisons between two or more people when each of them individually received gains or losses (Boksem, Kostermans, \& De Cremer, 2011; Luo et al., 2015; Qi, Wu, Raiha, \& Liu, 2018). For instance, in a two-person gambling game, Boksem et al. (2011) found that the FRN amplitude associated with deviations from the desired outcome significantly increased when an individual's own outcomes were worse than those of others. This finding suggests that in the human brain, whether an outcome is evaluated to be a success depends on the outcomes of others. Moreover, Luo et al. (2015) used a threeperson gambling game and found that the FRN amplitude was hierarchically sensitive to social comparison in the win condition but not in the loss condition. This finding indicates that there is a more negative-going weight on gains than on losses in social outcome evaluation. Qi et al. (2018) used a similar three-person gambling game and found that in the selfwin condition, the FRN exhibited a social comparison effect for both prosocial and proself individuals, while only prosocial individuals displayed this effect in the self-loss condition.

Other studies have explored participants receiving gains or losses together, but with different proportions of money (Qiu et al., 2010; Wu et al., 2012). They found that social comparison modulated not only the FRN, but also at a later stage of the outcome evaluation indexed by the P300. Specifically, equal payoffs and advantageous unequal payoffs elicited a larger P300 than disadvantageous unequal payoffs following gains (Qiu et al., 2010; Wu et al., 2012). In short, the social comparison effect on the FRN and P300 depends on an individual's own outcome, as people primarily show a downward comparison in the self-gain condition, but an upward comparison in the self-loss condition.

However, most previous studies investigated social comparison with unfamiliar persons, but few neuroscientific studies have considered social interactions involving familiar ones, such as relatives and friends. Behavioral studies have found that different kinds of interpersonal relationships affect people's self-evaluation in terms of social comparisons. Tesser, Millar, and Moore (1988) described the pain and pleasure of being close: During social comparison, when one is outperformed by others in a task highly relevant to self-esteem, a closer relationship between two persons results in a 
stronger threat to self-evaluation. In this study, we focus on whether interpersonal relationships also have a moderating effect on outcome evaluation in a social comparison context that unfolds over time.

\section{Interpersonal relationship}

In line with our research interest, previous ERP studies have discovered that vicarious learning is more effective with close others than with strangers. In addition, this effect was mediated by the degree to which participants incorporated close others in their self-concept (Kang, Hirsh, \& Chasteen, 2010; Leng \& Zhou, 2010). For instance, Kang et al. (2010) found that participants displayed a more negative-going FRN when observing mistakes made by friends as compared with strangers. Furthermore, Leng and Zhou (2010) found that a larger P300 emerged for a friend's outcomes as compared with a stranger's outcomes, while no FRN differences were observed between interpersonal conditions. In addition, Ma et al. (2011) showed that the P300 amplitude increased when the participant observed a difference in a friend's performance compared with a stranger regardless of whether he or she was involved in the game. Qi et al. (2018) examined electrophysiological correlates captured when individuals with different social value orientations engaged in a gambling game with two other players. The results showed that for prosocials only, winning with a socially liking player elicited an increased P300 compared with winning with a disliking player.

In terms of the competition context, Wang et al. (2014) asked participants to engage in an interactive gambling task against either a friend or a stranger. The P300 was modulated by feedback valence (gain or loss) and the nature of the interpersonal relationship, such that it was larger when competing against strangers. Finally, Chen, Lu, Wang, Feng, and Yuan (2017) used a chicken game and found that, when an opponent chose to cooperate, outcome feedback elicited a smaller FRN and a larger P300. The P300 was larger when participants were gaming with friends than when they were gaming with strangers. The difference of the P300 amplitude between the friend and the stranger conditions suggests that individuals have stronger motivations to win against strangers. Indeed, previous ERP studies have also found differences in evaluating the results of friends and strangers in other social interaction contexts (Campanha, Minati, Fregni, \& Boggio, 2011; Zhu et al., 2016).

In different kinds of social interaction contexts, interpersonal relationships may have different influences on outcome evaluation. Although previous studies have shown that brain potentials recorded from passive observers, cooperators, and competitors are different when gambling outcomes are delivered to a friend or a stranger. However, to our knowledge, previous studies have not yet considered the impact of interpersonal relationship on outcome evaluation in social comparison contexts. Investigating this topic could help interpret how social factors modulate the mechanisms of reward processing during social interaction, which is important to understand human social behavior and is the main interest of the current study. In contrast to Boksem et al. (2011), which used a simple time-estimation reaction-time task in which participants compared their performance with strangers (see below), this study used a more classical modified simple gambling paradigm (Gehring \& Willoughby, 2002; Hu, Xu, \& Mai, 2017b) and added a factor of interpersonal relationship (i.e., playing with a friend or a stranger). We are interested in the modulating effect of interpersonal relationships on outcome evaluation in a social comparison context, possibly manifesting on temporally sensitive ERP components.

\section{Overview}

To investigate this issue, we modified the simple gambling paradigm (Gehring \& Willoughby, 2002; Hu, Xu, et al., $2017 b$ ) to examine the neural effects of interpersonal relationship on outcome evaluation in a social comparison context. In line with previous ERP studies on outcome evaluation, we focused on the FRN and P300. We first identified participants' interpersonal relationships with the Inclusion of Others in the Self (IOS) scale (Aron, Aron, \& Smollan, 1992). Then, a simple monetary gambling task was used to explore whether the FRN and P300 in response to social outcomes would be influenced by different kinds of interpersonal relationships.

Boksem et al. (2011) reported that, when comparing with a stranger, FRN amplitudes were significantly more negative going in the other-gain condition than the other-loss condition in the self-loss condition. In contrast, in the self-gain condition, FRN amplitudes did not differ with regard to the outcome for the other player. Regarding the P300 component, the feedback indicating ones' correct performance resulted in a larger P300 compared with the feedback indicating his or her incorrect performance, but this pattern was reversed for others. We tested the replicability of these findings on the FRN and P300 and, more importantly, conducted an interpersonal relationships examination of the FRN and P300. In addition, we were interested in the differences in social comparisons between the self-gain condition and the self-loss condition, because people mainly show a downward comparison in the self-gain condition but an upward comparison in the self-loss condition (Boksem et al., 2011; Qi et al., 2018). We hypothesized that the relationship effects would regulate how we evaluate other people's outcomes in different self-states (as a function of the pain and pleasure of closeness), manifesting on the FRN and P300. We predicted that in the self-gain condition, people would prefer their friends to gain money, and in the self-loss condition, people would not prefer either their friends or 
strangers to gain money. Accordingly, the FRN amplitude would increase (i.e., more negative going) in response to a stranger's gain than a friend's gain in the self-gain condition, and the reverse would be true for the P300; meanwhile, the FRN and P300 would not distinguish between a friend's gain and a stranger's gain in the self-loss condition.

\section{Materials and methods}

\section{Participants}

Eighteen healthy students ( 10 females; mean age $21.39 \pm 1.58$ years) $(M \pm S D)$ from Liaoning Normal University were recruited as participants. We determined the sample size based on two considerations: (1) how many participants were usually involved in previous ERP studies (e.g., Wang et al., 2014; Weinberg et al., 2012; Weinberg, Riesel, \& Proudfit, 2014), and (2) how many participants were needed to ensure $80 \%$ statistical power to detect a small-to-medium effect size (Vazire, 2016). All participants reported normal or correctedto-normal vision and were right-handed, without cognitive or neurological impairments. None of the participants defined themselves as color blind. In order to control for the possibility that participants' perceived socioeconomic status might influence their economic choices in the experiments, all participants were carefully screened for their income levels before recruitment (Kang, Lee, Choi, \& Kim, 2013; Ly, Haynes, Barter, Weinberger, \& Zink, 2011). Only participants who reported midrange income levels (¥1,000 2,000, about US\$143 287 per month) were recruited in this study. This study was carried out in accordance with the Declaration of Helsinki, and written informed consent was obtained from all participants. The protocol was approved by the institutional ethics committee of the Liaoning Normal University.

\section{Questionnaires}

Participants were asked to bring a close friend to participate in the study with them. However, the level of friendship may have varied across individuals, which might be a confounding factor (Wang et al., 2014). In order to strictly control the quality of friendship, participants were required to complete the IOS questionnaire (Kang et al., 2010). The IOS is a one-item pictorial questionnaire, consisting of seven overlapping circles (one of which represents the participants themselves, and the other represents other people). The overlap of two circles increases linearly, forming a seven-point equidistant questionnaire: One point represents no overlap, and seven points represent almost complete overlap. Out of these seven options, participants select the one that represents the relationship between themselves and close others most appropriately (Aron et al., 1992). The IOS was completed twice by each participant: participants first reported the overlap between themselves and the friend that they brought to the lab, and then the overlap between themselves and the putative player (i.e., stranger).

\section{Experimental paradigm: Simple gambling task}

Participants were asked to bring a friend of the same gender to the lab. Participants decided who the ERP participant would be. A same-gender collaborator served as another putative player (i.e., stranger). The three participants received experimental guidance together, then the participant (i.e., ERP participant) who received the electroencephalogram (EEG) recording sat in a dim room, while his or her friend and the stranger sat in another room nearby. The formal experiment consisted of two conditions: In the first condition, the ERP participant finished the gambling task (see below) with the stranger; in the second condition, he or she finished the gambling task with the friend. At the beginning of each condition, the ERP participant was informed with whom they would conduct the task (i.e., friend or stranger). The sequence of the two conditions was counterbalanced between participants (see Fig. 1).

Before the experiment, the ERP participant was informed that the baseline payment was 60 Chinese yuan (about US\$9). The more money that each player earned for himself or herself during the task, the greater the bonus they would receive at the end of the ERP experiment. In other words, they were instructed to win as much money as possible. Participants were also informed that the outcomes provided to the other two players (i.e., friend and stranger) were determined independently of their own outcomes. Ultimately, they were paid

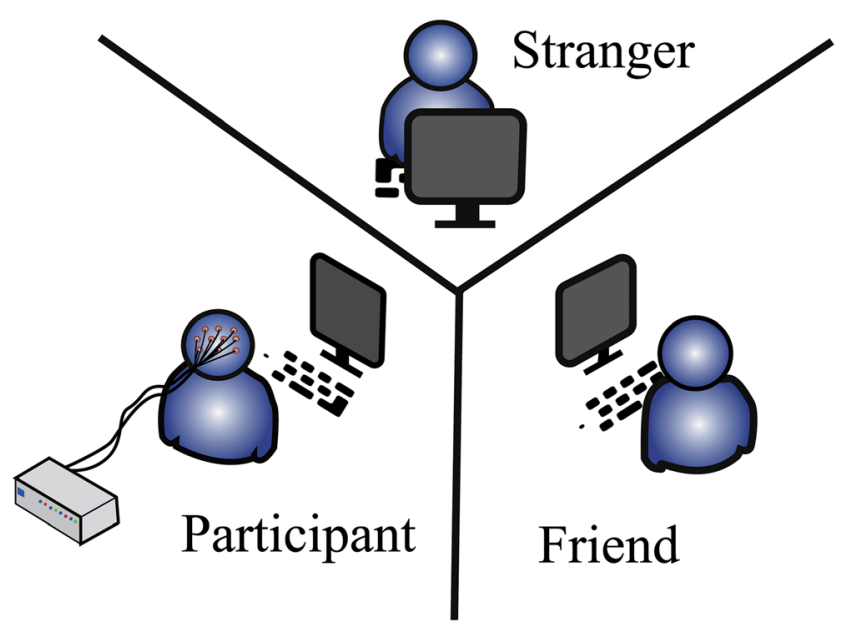

Fig. 1 Experimental schematic diagram. Three people participated in the lottery game, and only one of them had EEG data collected while performing the gambling task. At the beginning of each stage, the ERP participant was informed whom they would conduct the task with (i.e., friend or stranger). The sequence of the two stages was counterbalanced between the participants 
with an amount of money between 60 and 70 yuan (about US\$9 10).

We used a simple gambling task (Fig. 2) modified from a previous study (Hu, Xu, et al., 2017b). During the gambling task, the ERP participant sat in an electrically shielded room approximately $75 \mathrm{~cm}$ from a 19-inch LCD computer monitor (refresh rate: $60 \mathrm{~Hz}$; resolution: $1,440 \times 900$ pixels). As illustrated in Fig. 2, each trial began with a white fixation cross presented for $500 \mathrm{~ms}$ on a black background. Afterward, two gray squares $\left(1.9^{\circ} \times 1.9^{\circ}\right.$ of visual angle) representing two options appeared on the left and right side of the fixation point. Participants were required to choose the left or right square by pressing the "F" or "J" key on the keyboard with their left or right index finger, respectively. The chosen option was highlighted by a thickening of the red border for $500 \mathrm{~ms}$. After an interval of 500-1,000 ms, the outcomes feedback corresponding to the chosen option for the ERP participant and the other player (friend or stranger) were displayed on two squares, respectively, in a horizontal array for 1,000 ms. In terms of its valence, the outcome was either a gain (indicated by the symbol "+") or a loss (indicated by the symbol “-”). The ERP participant's own outcome was presented in the square with a red border, and the other's outcome was presented in the square with a blue border. The horizontal positions (left/right) of the two squares were randomized across trials. It must be noted that compared with the classic simple gambling paradigm (Gehring \& Willoughby, 2002), the current study includes a scenario of social comparison. Specifically, two players played the simple gambling paradigm game at the same time and their results were presented simultaneously. However, the outcome of one another was unrelated.

From the perspective of the ERP participants, there were four kinds of possible outcomes in the gambling task: selfgain and other-loss (hereinafter labeled as "GL"), self-gain and other-gain ("GG"), self-loss and other-loss ("LL"), as well as self-loss and other-gain ("LG"). The intertrial interval lasted for $500 \mathrm{~ms}$, and all conditions differed only in terms of outcome valence; the outcome magnitude was kept constant across conditions. In the condition of "self-gain and other-loss" (+ and -, GL), the ERP participant gained 0.5 yuan, while the other player (friend or stranger) lost 0.5 yuan; in the "both-gain condition" ( + and,$+ \mathrm{GG})$, both players gained 0.5 yuan; in the "both-loss condition" (- and -, LL), both players lost 0.5 yuan; in the "self-loss and other-gain condition" (and,$+ \mathrm{LG}$ ), the ERP participant lost 0.5 yuan, while the other player gained 0.5 yuan. Unknown to the participants, all kinds of outcome feedback were presented randomly, and each participant received equal numbers of each kind of feedback throughout the task.

Before the experiment, participants practiced the task for 12 trials. In the formal task, both the friend condition and the stranger condition consisted of 320 trials dividing into eight blocks, such that each feedback condition (GL/GG/LL/LG) involved 80 trials for the friend or for the stranger. Accordingly, the whole task involved 640 trials and lasted for approximately 50-60 min. Upon finishing the experiment, all participants were queried about the credibility of the stranger scenario, and no one raised any doubts. In addition, before the experiment, participants answered the IOS questionnaire. After the experiment, participants rated their level of satisfaction on a 7-point scale $(1=$ very dissatisfied; $7=$ very satisfied $)$ for each type of feedback. The stimulus presentation and the behavioral data acquisition were conducted with E-Prime 2.0 software (PST, Inc., Pittsburgh, PA, USA).

\section{EEG recording and analyses}

EEG signals were recorded by a 64-channel amplifier $(500 \mathrm{~Hz}$ sampling rate, ANT Neuro EEGO Inc., Germany) using an elastic cap, containing 64 unshielded and sintered $\mathrm{Ag} / \mathrm{AgCl}$ electrodes, with the electrode layout conforming to the International 10-20 electrode system. For monitoring ocular movements and eye blinks, electrooculogram (EOG) recordings were taken from four electrodes placed lateral to each eye

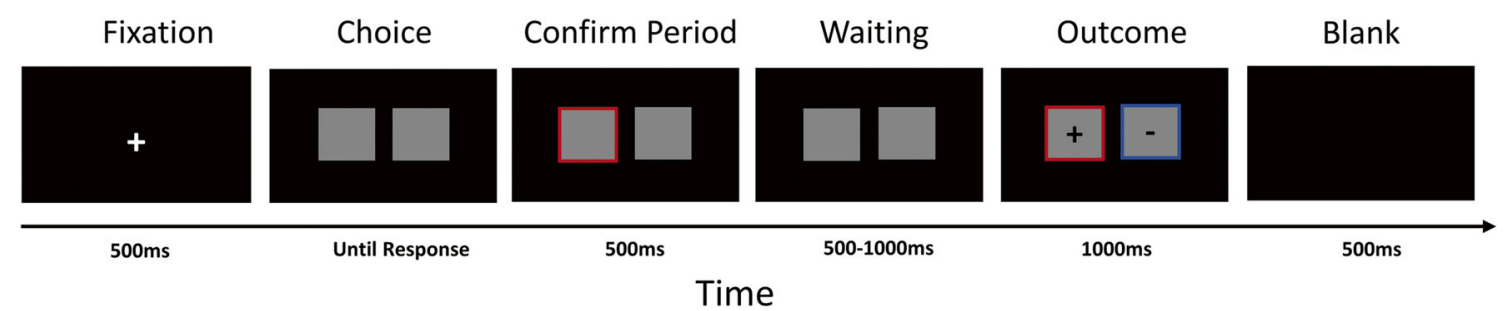

Fig. 2 An illustration of a single trial in the gambling task. Each trial began with a fixation cross. Participants viewed two gray squares (indicating two options) and were required to select one of the squares by pressing a corresponding key ( $\mathrm{F}$ for the left square and $\mathrm{J}$ for the right). Their choice was then highlighted for $500 \mathrm{~ms}$. After an interval of 500$1,000 \mathrm{~ms}$, the outcome feedback was presented for $1,000 \mathrm{~ms}$, with participant's own outcome displayed in the square with the red border

and the others outcomes displayed in the square with the blue border. There were four possible outcomes probabilities, resulting in four experimental conditions. Participants were instructed that there was no relationship between the location of cards and outcomes, and that the consequences of his or her decisions would not affect the consequences of other players' choices or scores, and vice versa. (Color figure online) 
and above and below the right eye. Electrode impedance was always kept below $10 \mathrm{k} \Omega$.

Analyzer 2.0 software (Brain Products, Munich, Germany) was used to analyze the off-line EEG data. Data were rereferenced off-line to the average of the left and right mastoids. Major artifacts, such as ocular movement, eyeblinks, and muscle-related potentials, were corrected with an independent component analysis (Jung et al., 2000). Then, the data were digitally filtered at a low-pass $30 \mathrm{~Hz}(24 \mathrm{~dB} /$ octave) and segmented from $200 \mathrm{~ms}$ before to $1,000 \mathrm{~ms}$ after stimulus onset. After baseline correction ( -200 to $0 \mathrm{~ms}$ ), trials with amplitudes exceeding $\pm 80 \mu \mathrm{V}$ at any electrode were excluded from the average in order to eliminate the contamination of larger artifacts. After artifact rejection, feedback conditions contained an average of 77 trials for GL (min: 70/max: 80), 76 trials for GG (min: 63/max: 80), 76 trials for LL (min: 65/ max: 80), and 76 trials for $L G$ (min: 66/max: 80) when playing with a stranger, and an average of 76 trials for GL (min: 61/ max: 80), 76 trials for GG (min: 64/max: 80), 76 trials for LL (min: 64/max: 80), and 74 trials for LG (min: 58/max: 80) when playing with a friend. Due to the relatively small sample size, we analyzed the internal consistency reliability of each conditions, and found that the reliability value of each condition was greater than 0.7 . This result showed that the internal consistency of each condition was relatively high and that the results were reliable.

For the FRN, we measured the mean amplitudes in the time window of 250-340 ms; for the P300, we measured the mean amplitudes in the time window of $340-470 \mathrm{~ms}$. In preliminary analyses, the anteroposterior and lateral scalp locations were considered as topographic factors. Based on the topographical distribution of each ERP component (see Figs. 4 and 5) and previous studies (Gehring \& Willoughby, 2002; Yeung \& Sanfey, 2004), the FRN was calculated across nine electrode locations (Fz, F3, F4, FC3, C3, FCz, Cz, FC4, and C4), and the $\mathrm{P} 300$ was quantified across nine electrode locations ( $\mathrm{C} 3$, $\mathrm{CP} 3, \mathrm{P} 3, \mathrm{Cz}, \mathrm{CPz}, \mathrm{Pz}, \mathrm{C} 4, \mathrm{CP} 4$, and P4) that were chosen to cover scalp areas corresponding with these components. Among the nine electrode locations F3, F4, Fz, FC3, C3, $\mathrm{FCz}, \mathrm{Cz}, \mathrm{FC} 4$, and $\mathrm{C} 4$, the results of descriptive statistics showed that $\mathrm{FCz}$ had the largest FRN amplitude. Meanwhile, among the nine electrode locations C3, CP3, P3, Cz, CPz, Pz, C4, CP4, and P4, CPz had the largest P300 amplitude. Hence, we focused on the $\mathrm{FCz}$ and $\mathrm{CPz}$ sites for detailed analyses, as the ERP amplitudes on these sites were maximal.

Behavioral and ERP data were statistically analyzed using SPSS software (Version 22.0, SPSS Inc., Chicago, IL, USA). The FRN amplitude, P300 amplitude, and satisfaction scores were analyzed separately using a three-way repeated measure ANOVA of 2 (interpersonal relationships: stranger vs. friend) $\times 2$ (self-outcome: self-gain vs. self-loss) $\times 2$ (other-outcome: other-gain vs. other-loss). All of them were within-subject factors. The significance level was set at 0.05 for all the analyses in the present study. The Greenhouse-Geisser correction was conducted to account for sphericity violations whenever appropriate. Post hoc testing of the significant main effects was applied using Bonferroni adjustments. For convenience, in the following description, the relationship effect refers to the difference between friends and strangers. The selfoutcome effect means the difference between one's own wins and losses. The other-outcome effect refers to the difference between the other player's wins and losses.

\section{Results}

\section{Questionnaire results}

The analysis of IOS scores reported by the ERP participants revealed that the relationship between the participant and friend $(5.06 \pm 0.26)(M \pm S E)$ was much closer than with the stranger $(1.83 \pm 0.15), t(17)=-11.24, p<.001$, Cohen's $d=$ 2.69. This result shows that the interpersonal relationship between friends and strangers was significantly different.

For the satisfaction scores reported by the ERP participants, the main effect of the self-outcome was significant, $F(1,17)=$ $234.38, p<.001, \eta 2 \mathrm{p}=.93$, indicating that the satisfaction score was larger when participants received a gain $(5.67 \pm$ $0.16)(M \pm S E)$ than a loss $(3.30 \pm 0.16)$. Furthermore, the main effect of the other-outcome was significant, $F(1,17)=$ $6.34, p=.02, \eta 2 \mathrm{p}=.27$, indicating that the satisfaction score was larger when the other player received a gain $(4.76 \pm 0.17)$ than a loss $(4.15 \pm 0.20)$. The results of the repeated measure ANOVAs are shown in Fig. 3.

Importantly, a significant Relationship $\times$ Other Outcome interaction effect was observed, $F(1,17)=12.61, p=.002$, $\eta 2 p=.43$. A further simple effect analysis showed that the relationship effect in the other-gain condition was significant, $F(1,17)=15.42, p=.001, \eta 2 p=.47$, such that the satisfaction score was larger for the friend's gain $(5.11 \pm 0.17)$ than for the stranger' gain (4.41 \pm 0.19$)$. In contrast, the relationship effect was not significant in the other-loss condition, $F(1,17)=3.34$, $p=.08, \eta 2 p=.16$.

A significant Self-Outcome $\times$ Other-Outcome interaction effect was also observed, $F(1,17)=15.32, p=.001, \eta 2 \mathrm{p}=$ .47. Consequently, a simple effect analysis was applied to this interaction. The results showed that the other-outcome effect in the self-gain condition was significant, $F(1,17)=19.00, p<$ $.001, \eta 2 \mathrm{p}=.53$, such that satisfaction scores were larger in GG $(6.19 \pm 0.17)$ than GL $(5.14 \pm 0.23)$. Contrastingly, the other-outcome effect was not significant in the self-loss condition, $F(1,17)=0.33, p=.58, \eta 2 \mathrm{p}=.02$.

In addition, the main effect of this relationship was not significant, $F(1,17)=0.09, p=.77, \eta 2 \mathrm{p}=.01$, and no significant interaction effects were observed for the 


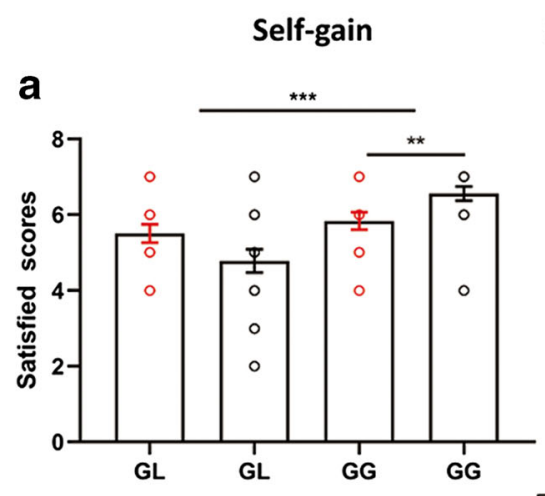

Satisfied Scores

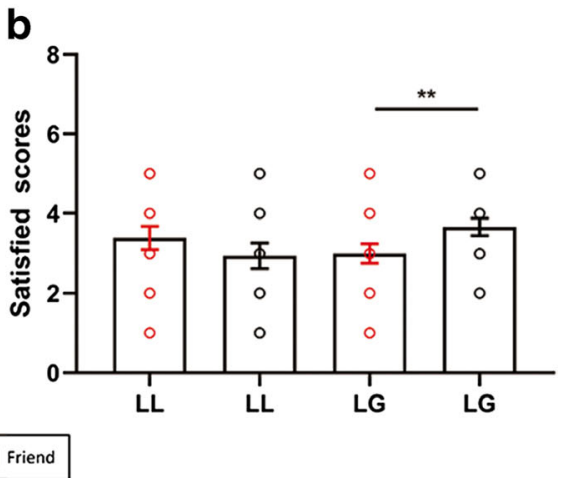

Fig. 3 a The bar graphs shown the mean values of the satisfaction scores of the relationship effect were different in the other-gain condition and other-loss condition. b The bar graphs shown the mean value of the

Relationship $\times$ Self-Outcome, $F(1,17)=0.26, p=.62, \eta 2 \mathrm{p}=$ .02 , and Relationship $\times$ Self-Outcome $\times$ Other-Outcome, $F(1$, $17)=0.49, p=.50, \eta 2 \mathrm{p}=.03$.

\section{ERP results}

FRN For the FRN amplitude, the results of the repeatedmeasure ANOVAs are shown in Fig. 4. The main effect of self-outcome was significant, $F(1,17)=21.03, p<.001, \eta 2 \mathrm{p}$ $=.55$, indicating that the FRN was more negative going (negative polarity: larger voltages indicate smaller amplitudes) when participants received a loss $(3.73 \pm 0.87 \mu \mathrm{V})$ than a when they received a gain $(6.29 \pm 1.27 \mu \mathrm{V})$. Yet the main effects of the other-outcome, $F(1,17)=0.01, p=.91, \eta 2 \mathrm{p}$ $=.001$, and relationship, $F(1,17)=0.11, p=.74, \eta 2 \mathrm{p}=.006$, were not significant. On the other hand, there were significant interaction effects observed on Self-Outcome $\times$ OtherOutcome, $F(1,17)=16.14, p=.001, \eta 2 \mathrm{p}=.49$, and Relationship $\times$ Self-Outcome $\times$ Other-Outcome, $F(1,17)=$ $6.16, p=.02, \eta 2 \mathrm{p}=.27$ ).

A simple effect analysis was conducted to investigate the Self-Outcome $\times$ Other-Outcome interaction. The results revealed that in the self-gain condition, the other-outcome effect of FRN was significant, $F(1,17)=7.60, p=.01, \eta 2 \mathrm{p}=.30$, such that the FRN was more negative going when the other player received a loss $(5.85 \pm 1.22 \mu \mathrm{V})$ compared with when they received a gain $(6.71 \pm 1.32 \mu \mathrm{V})$. In contrast, in the selfloss condition, the other-outcome effect of FRN was significant, $F(1,17)=8.38, p=.01, \eta 2 \mathrm{p}=.33$, but the FRN was more negative going when the other player received a gain $(3.32 \pm 0.86 \mu \mathrm{V})$ as opposed to a loss $(4.13 \pm 0.89 \mu \mathrm{V})$.

A simple effect analysis was conducted to investigate the three-way Relationship $\times$ Self-Outcome $\times$ Other-Outcome interaction. The results revealed that the relationship effect of the FRN was only significant in the GG condition, $F(1,17)=$ $4.78, p=.04, \eta 2 \mathrm{p}=.22$. The FRN was more negative going satisfaction scores for other-outcomes was different in the self-gain condition and self-loss condition. Error bars indicate the standard error of the mean $(S E M) . * * p<.01 ., * * * p<.001$

when a stranger gained $(6.23 \pm 1.39 \mu \mathrm{V})$ than when a friend and ERP participant $(7.18 \pm 1.29 \mu \mathrm{V})$ both gained (please see Fig. 4).

P300 For the P300 amplitude, the results analyzed by the repeated-measure ANOVAs are shown in Fig. 5. The main effect of self-outcome was significant, $F(1,17)=11.55, p=$ $.003, \eta \mathrm{p} 2=.41$, indicating that the P300 was larger when participants received a gain $(8.47 \pm 1.36 \mu \mathrm{V})$ than a loss $(7.69 \pm 1.40 \mu \mathrm{V})$. The main effect of other-outcome was significant, $F(1,17)=8.66, p=.009, \eta 2 p=.34$, suggesting that the P300 was generally larger in the case that the other player gains $(8.42 \pm 1.43 \mu \mathrm{V})$ compared with a loss $(7.74 \pm 1.33 \mu \mathrm{V})$. In addition, the main effect of relationship was not significant, $F(1,17)=0.34, p=.57, \eta 2 p=.02$, and there were no significant interaction effects on the Relationship $\times$ Self-Outcomes, $F(1,17)=4.40, p=.05, \eta 2 p=.21$, the Relationship $\times$ OtherOutcome, $F(1,17)=0.03, p=.86, \eta 2 p=.002$, Self-Outcome $\times$ Other-Outcome, $F(1,17)=0.09, p=.77, \eta 2 p=.005$, and Relationship $\times$ Self-Outcome $\times$ Other-Outcome interaction terms, $F(1,17)=1.76, p=.20, \eta 2 p=.09$ (see Fig. 5).

\section{Discussion}

Based on previous research, the current study examined the effect of interpersonal relationships on social comparison. On the behavioral level, the satisfaction score was sensitive to other player's outcomes only when participants themselves received a gain. Furthermore, the satisfaction score was sensitive to other's outcome only when participants compared themselves with a friend as opposed to a stranger. On the ERP level, we observed a social comparison effect and an effect of interpersonal relationship on the FRN in certain conditions. In contrast, the P300 only registered participants' attention resource allocation toward monetary gains for 


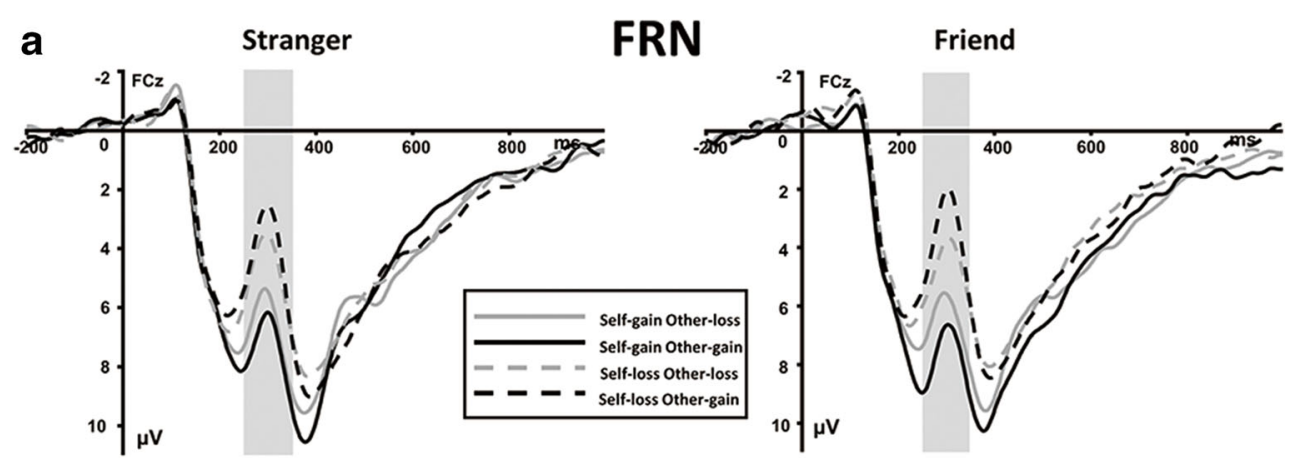

b

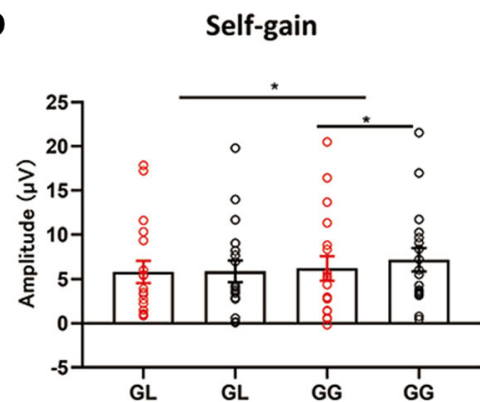

\section{FRN}

Self-loss

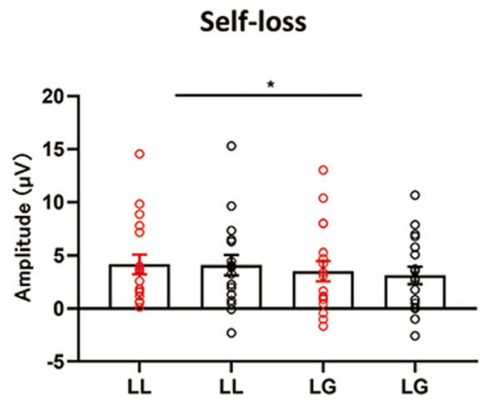

Stranger Friend

C

\section{Other-loss minus Other-gain}

Stranger

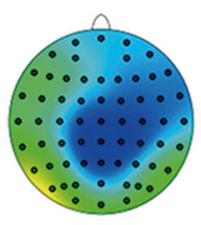

Self-gain

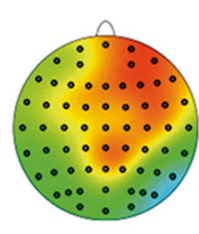

Self-loss

Fig. 4 a Grand-average ERP waveforms from the FCz electrode site. The gray areas highlight the time window of the FRN (250-340 ms) used for statistical analysis. b The bar graphs show the mean value of the FRN amplitude for each condition. Error bars indicate the standard error of the

themselves and for others, which was unaffected by the nature of the interpersonal relationship (friend/stranger). These findings are discussed below.

The FRN results showed that outcome evaluation is heavily influenced by social comparisons. The FRN is typically more negative going for negative outcomes than for positive ones in nonsocial situations, regardless of outcome magnitude (Hajcak, Moser, Holroyd, \& Simons, 2006). Consistent with this knowledge, the present study found that the FRN was more negative going when participants received a loss in contrast to a gain. Meanwhile, the main effect of other-outcome was not significant in this study. This is also consistent with previous findings that FRN amplitudes were indifferent to other people's monetary gains and losses when participants

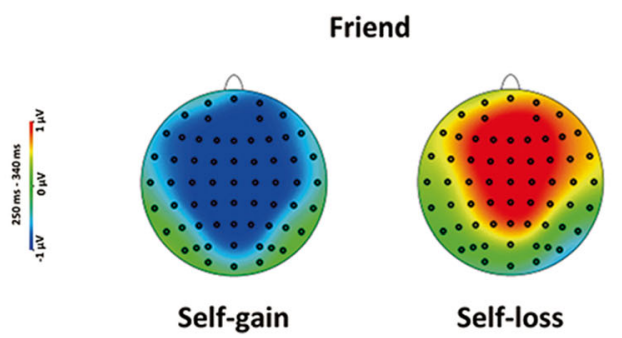

mean (SEM). c Topographical voltage distributions of the other outcome's d-FRN for each condition (left row for the stranger and right row for the friend). $* p<.05$

themselves were involved in the task (Ma et al., 2011). As described in the introduction, when individuals are directly involved in a task, they are mostly concerned with their own payoffs (Leng \& Zhou, 2014; Marco-Pallares, Kramer, Strehl, Schroder, \& Munte, 2010).

Boksem et al. (2011) showed that in response to negative feedback, FRN amplitudes were significantly more negative going when the other player experienced a gain, compared with when the other player experienced a loss. In the current study, we also found that in the self-loss condition, the FRN was more negative going when the other player received a gain than a loss. We speculated that although the results for two players were independent from one another, for ERP participants, another player in the self-loss condition served as an 

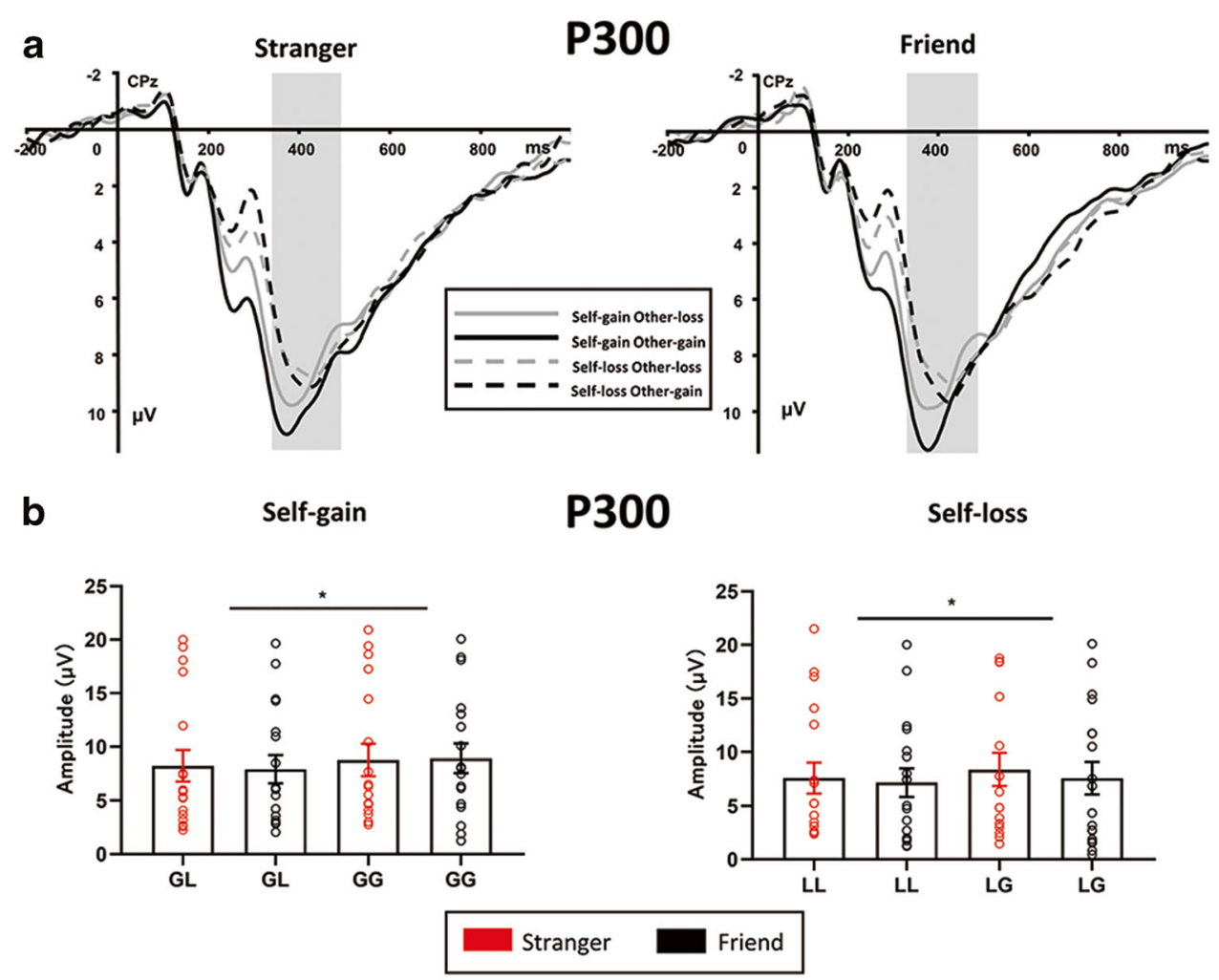

C

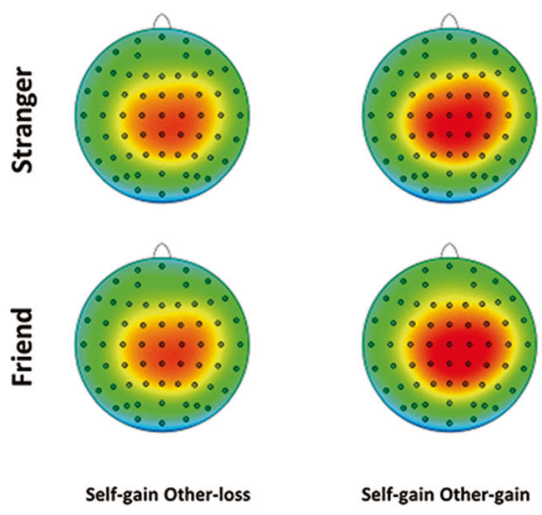

Fig. 5 a Grand-average ERP waveforms from the CPz electrode site. The gray areas highlight the time window of the P300 (340-470 ms) used for the statistical analysis. $\mathbf{b}$ The bar graphs show the mean value of the P300 amplitude for each condition. Error bars indicate the standard error of the

interest-relevant competitor (Fukushima \& Hiraki, 2006; Wang et al., 2017). Accordingly, monetary losses for oneself may have turned into a threat to self-esteem in the context of social comparison, and thus led to a more negative-going FRN when others gained. Yet the FRN pattern was reversed in the self-gain condition - that is, in the self-gain condition, the FRN was more negative going when the other player received a loss compared with a gain. As described in the introduction, the FRN is sensitive to expectancy violation. In our opinion, the current result indicates that when participants received a gain, they also expected others to win (which was in line with the satisfaction score results). Boksem et al. (2011) found that

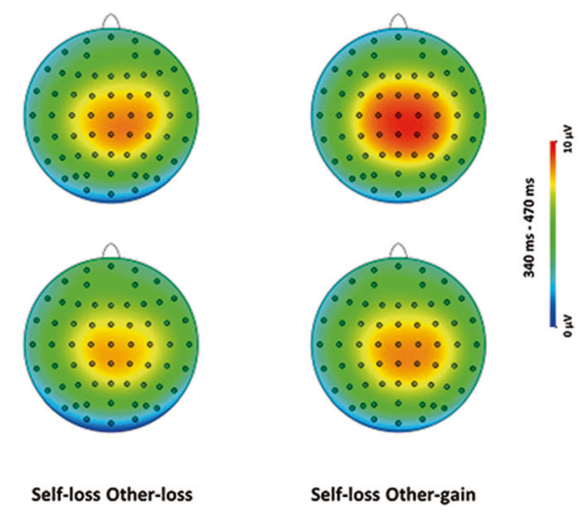

mean (SEM). c Topographical voltage distributions of the P300 for each condition are separated by relationships (top row for the stranger and bottom row for the friend)

FRN amplitudes did not differ according to the outcomes for the others in the self-gain condition. Nevertheless, in the current study, we found that the opposite was true in the self-gain condition.

To explain this inconsistency, we propose that cultural differences should be taken into account. Varnum, Shi, Chen, Qiu, and Han (2014) point out that Chinese participants reported similar levels of feeling happy in response to self-win and friend-win, but they felt less happy in response to self-loss compared with friend-loss. Li et al. (2013) also reported that Chinese participants believed that team success was more important than their own performance. These phenomena were 
possibly because Chinese people tend to identify themselves as group members and are strongly motivated to behave according to group norms (Morris \& Peng, 1994). These results might reveal that people are more empathetic and cooperative to others when they are relatively successful (see also Shen et al., 2013), but are less so when their performance should be improved and social standing is threatened (see also Boksem et al., 2011).

Interestingly, the satisfaction scores and the FRN both showed that interpersonal relationships selectively modulate outcome evaluations in the social comparison context. The satisfaction scores showed that the relationship effect was significant only in the other-gain condition, such that the satisfaction score was larger for a friend's gain than a stranger's gain. The relationship effect on the ERP results were only found to be significant in the GG (self-gain and other-gain) condition, such that the FRN was more negative-going for friend-gain over stranger-gain. The present study found that the relationship effect on the FRN emerged in specific situations. This might suggest that whether outcome evaluation was sensitive to interpersonal relationship type depends on outcome valence. Shen et al. (2013) found that the high-pay group showed a more pronounced gain-loss disparity in the FRN amplitude toward others than participants themselves. However, this response pattern was not observed among participants in the low-pay group. Higher status might have enhanced participants' empathic responses toward others' outcomes, such that the effect of interpersonal relationship was more likely to emerge. That is, people exert more motivational relevance toward their friends than strangers, but this difference is salient only when they personally receive positive feedback. In general, these results show that interpersonal relationship selectively regulates outcome evaluation in the social comparison context, manifesting on the FRN. When people gain money, they prefer their friends rather than strangers to gain money; but when people lost money, they do not want another player to gain money, regardless of whether that player is a friend or a stranger.

The P300 is generally considered to reflect attentional resource allocation in outcome evaluation (Gray, Ambady, Lowenthal, \& Deldin, 2004). In the present study, the P300 was larger when participants received gains than losses, consistent with previous findings (Qi et al., 2018; Schreuders et al., 2018). In addition, the P300 amplitude difference between other's gains and other's losses showed similar pattern on self-outcomes. Baker and Holroyd (2011) reported that when participants predicted the other player to be more successful than the reality, there was a larger P300 and a stronger orienting response to observed failures of the other player. The discrepancy between the current P300 results and that of Baker and Holroyd (2011) may indicate the influence of cultural difference: While the self-interest bias dominates in Western societies, Chinese people attend to themselves and to other people at a similar level ( $\mathrm{Li}$ et al., 2013). However, this interpretation is speculative at this point. Future research should replicate our task design with a sample of individuals from different cultural backgrounds.

Inconsistent with our prior hypotheses, the P300 amplitude differences between different kinds of interpersonal relationships (friend vs. stranger) was not significant. The ERP analysis showed that, regardless of the interpersonal relationship type, the P300 was larger when either the ERP participant or the other player received a gain compared with a loss. This contradicts previous research, which has found that gaming with strangers evoked a larger P300 amplitude than gaming with friends (Chen et al., 2017). Possibly, this was due to the simplicity of the current task design. Specifically, Chen et al. (2017) asked participants to complete the chicken game with a friend or stranger, where participants needed to select a cooperative or aggressive decision depending on other's decision. In contrast, the two players in our task performed independently, and participants' decisions (as well as outcomes) would not affect their partners' decisions and vice versa. Consequently, the effect of interpersonal relationship appears at an earlier stage (indexed by the FRN) rather than a later stage (indexed by the P300) of outcome processing, regarding that social comparison could be completed rapidly in our simple task without deliberate thinking. This interpretation, however, remains speculative. Future research could distinguish between early and late processing by manipulating the difficulty level of the task.

The present study has a few major limitations. First, the results of traditional analysis of FRN may have been affected by temporal overlap between the FRN and P300 (Zhang et al., 2013). To address this issue, we used the principal component analysis (PCA) approach. The results of PCA have shown the effect of interpersonal relationship when the self gained money. Specifically, when participants gained money, the amplitude of the PCA factor (corresponding to the FRN in the conventionally averaged ERPs, but a more positivity-going means a larger amplitude, which is different from the FRN) was more positive going when a friend received a gain compared with a loss, while there is no difference between gains and losses for a stranger. In other words, when self gained money, we were more likely to expect our friends to get money, but we were not concerned about the results of the stranger. However, when self lost money, there are no difference in the interpersonal relationship effects between the different conditions (for details, see the Supplementary Materials). The findings of PCA in the present study are consistent with that in recent studies, which suggested that the variation in FRN amplitudes stemmed from neural activity associated with positive rather than negative feedback (Baker \& Holroyd, 2011; Foti, Kotov, 
Klein, \& Hajcak, 2011a; Holroyd, Krigolson, \& Lee, 2011). In a word, the interpersonal effect may have been mainly come from paying more attention to friends when the self gained money.

Second, all the participants were Chinese people, and we suggest follow-up researchers to recruit samples from other cultures (White \& Lehman, 2005). This is particularly important regarding that the current results might have been influenced by cultural differences, which have been addressed above. Specifically, individuals in interdependent cultures (e.g., East Asia) that emphasize maintaining social relationships with others are more likely to seek social comparison compared with individuals in independent cultures (e.g., North America) that emphasize one's internal feelings and thoughts (Guimond et al., 2007; P. Kang et al., 2013). As such, replicating the current study on Western participants would help to reveal whether social comparisons between different kinds of interpersonal relationships are sensitive to cultural variation.

Third, although many ERP studies focusing on social comparison have been conducted by presenting the results of self and others simultaneously (Boksem et al., 2011; Luo et al., 2015; Qiu et al., 2010), using this method holds the risk to influence the interpretation of the ERP findings. Presenting the self-related and other-related outcomes separately may lead to more rigorous results. Finally, we have only compared friends and strangers in the present study, but other types of relationships (e.g., romantic partners vs. parents) may also matter in terms of evaluating feedback in a social comparison context (Zhu et al., 2016). For example, intimacy affects bottom-up information processing involved in empathy, indicated by a positive correlation between the relationship level with participants' beloved ones and the deactivation level of the right temporoparietal junction (Cheng, Chen, Lin, Chou, \& Decety, 2010).

To sum up, this study indicates that the human brain evaluates outcomes in a social context-dependent pattern, as reflected by behavioral results and ERP signals. Specifically, the P300 registered participants' attentional resource allocation toward positive outcomes for the self and for others, regardless of the type of interpersonal relationships. Interestingly, the social comparison effect and the interpersonal relationship effect selectively manifested in the early stage of outcome processing, as indexed by the FRN. From an evolutionary perspective, we suggest that it is important to compare one's outcomes with that of others rapidly while also taking the interpersonal relationship context into account. This function helps us and significant others to survive and reproduce (Boksem et al., 2011). The similarity hypothesis of social comparison proposes that we compare ourselves with people around us, such as classmates or colleagues (Festinger, 1954). Most previous ERP studies, however, have only considered comparing oneself with a stranger. In this regard, our findings have extended the current knowledge on the temporal processing of social comparisons under different kinds of interpersonal relationships.

Open practices statement The data and materials for all experiments are available online (all results are provided at https://osf.io/m $4 \mathrm{~h} 7 \mathrm{~s} /$ ), and none of the experiments was preregistered.

Author contributions Z.H.Y. designed experiments; Z.H.Y., Z.L.L., and Z.D.F. carried out experiments; Z.H.Y. and L.J.C. analyzed experimental results; Z.H.Y., Z.M.M., L.J.C. wrote the manuscript; G.R.L. and L.W.B. provided critical review, commentary or revision and oversight.

Funding information This work was supported by the National Natural Science Foundation of China [31871106, 31571124] and Major Program of the Chinese National Social Science Foundation [17ZDA324].

\section{References}

Appel, H., Gerlach, A. L., \& Crusius, J. (2016). The interplay between Facebook use, social comparison, envy, and depression. Current Opinion in Psychology, 9, 44-49. doi:https://doi.org/10.1016/j. copsyc.2015.10.006

Aron, A., Aron, E. N., \& Smollan, D. (1992). Inclusion of other in the self scale and the structure of interpersonal closeness. Journal of Personality and Social Psychology, 63(4), 596-612. doi:https:// doi.org/10.1037/0022-3514.63.4.596

Baker, T. E., \& Holroyd, C. B. (2011). Dissociated roles of the anterior cingulate cortex in reward and conflict processing as revealed by the feedback error-related negativity and N200. Biological Psychology, 87(1), 25-34. doi:https://doi.org/10.1016/j.biopsycho.2011.01.010

Ball, R., \& Chernova, K. (2008). Absolute income, relative income, and happiness. Social Indicators Research, 88(3), 497-529. doi:https:// doi.org/10.1007/s11205-007-9217-0

Boksem, M. A. S., Kostermans, E., \& De Cremer, D. (2011). Failing where others have succeeded: Medial frontal negativity tracks failure in a social context. Psychophysiology, 48(7), 973-979. doi: https://doi.org/10.1111/j.1469-8986.2010.01163.x

Campanha, C., Minati, L., Fregni, F., \& Boggio, P. S. (2011). Responding to unfair offers made by a friend: Neuroelectrical activity changes in the anterior medial prefrontal cortex. Journal of Neuroscience, 31(43), 15569-15574. doi:https://doi.org/10.1523/jneurosci.125311.2011

Chen, J., Wu, Y., Tong, G. Y., Guan, X. M., \& Zhou, X. L. (2012). ERP correlates of social conformity in a line judgment task. $B M C$ Neuroscience, 13, 43. doi:https://doi.org/10.1186/1471-2202-13-43

Chen, Y. Z., Lu, J. M., Wang, Y. W., Feng, Z. Q., \& Yuan, B. (2017). Social distance influences the outcome evaluation of cooperation and conflict: Evidence from event-related potentials. Neuroscience Letters, 647, 78-84. doi:https://doi.org/10.1016/j.neulet.2017.03. 018

Cheng, Y., Chen, C., Lin, C., Chou, K. H., \& Decety, J. (2010). Love hurts: An fMRI study. NeuroImage, 51(2), 923-929. doi:https://doi. org/10.1016/j.neuroimage.2010.02.047

Dvash, J., Gilam, G., Ben-Ze'ev, A., Hendler, T., \& Shamay-Tsoory, S. G. (2010). The envious brain: The neural basis of social comparison. Human Brain Mapping, 31(11), 1741-1750. doi:https://doi.org/10. 1002/hbm.20972 
Engelmann, J. B., \& Pessoa, L. (2007). Motivation sharpens exogenous spatial attention. Emotion, 7(3), 668-674. doi:https://doi.org/10. 1037/1528-3542.7.3.668

Festinger, L. (1954). A theory of social comparison processes. Human Relations, 7(7), 117-140. doi:https://doi.org/10.1177/ 001872675400700202

Fliessbach, K., Weber, B., Trautner, P., Dohmen, T., Sunde, U., Elger, C. E., \& Falk, A. (2007). Social comparison affects reward-related brain activity in the human ventral striatum. Science, 318(5854), 1305-1308. doi:https://doi.org/10.1126/science.1145876

Foti, D., Kotov, R., Klein, D. N., \& Hajcak, G. (2011a). Abnormal neural sensitivity to monetary gains versus losses among adolescents at risk for depression. Journal of Abnormal Child Psychology, 39(7), 913924. doi:https://doi.org/10.1007/s10802-011-9503-9

Foti, D., Weinberg, A., Dien, J., \& Hajcak, G. (2011b). Event-related potential activity in the basal ganglia differentiates rewards from nonrewards: Temporospatial principal components analysis and source localization of the feedback negativity. Human Brain Mapping, 32(12), 2207-2216. doi:https://doi.org/10.1002/hbm. 21182

Fukushima, H., \& Hiraki, K. (2006). Perceiving an opponent's loss: Gender-related differences in the medial-frontal negativity. Social Cognitive and Affective Neuroscience, 1(2), 149-157. doi:https:// doi.org/10.1093/scan/ns1020

Gehring, W. J., \& Willoughby, A. R. (2002). The medial frontal cortex and the rapid processing of monetary gains and losses. Science, 295(5563), 2279-2282. doi:https://doi.org/10.1126/science. 1066893

Gray, H. M., Ambady, N., Lowenthal, W. T., \& Deldin, P. (2004). P300 as an index of attention to self-relevant stimuli. Journal of Experimental Social Psychology, 40(2), 216-224. doi:https://doi. org/10.1016/s0022-1031(03)00092-1

Gu, R. L., Zhang, D. D., Luo, Y., Wang, H. Y., \& Broster, L. S. (2018). Predicting risk decisions in a modified Balloon Analogue Risk Task: Conventional and single-trial ERP analyses. Cognitive Affective \& Behavioral Neuroscience, 18(1), 99-116. doi:https://doi.org/10. 3758/s13415-017-0555-3

Guimond, S., Branscombe, N. R., Brunot, S., Buunk, A. P., Chatard, A., Desert, M., ... Yzerbyt, V. (2007). Culture, gender, and the self: Variations and impact of social comparison processes. Journal of Personality and Social Psychology, 92(6), 1118-1134. doi:https:// doi.org/10.1037/0022-3514.92.6.1118

Hajcak, G., Holroyd, C. B., Moser, J. S., \& Simons, R. F. (2005). Brain potentials associated with expected and unexpected good and bad outcomes. Psychophysiology, 42(2), 161-170. doi:https://doi.org/ 10.1111/j.1469-8986.2005.00278.x

Hajcak, G., Moser, J. S., Holroyd, C. B., \& Simons, R. F. (2006). The feedback-related negativity reflects the binary evaluation of good versus bad outcomes. Biological Psychology, 71(2), 148-154. doi: https://doi.org/10.1016/j.biopsycho.2005.04.001

Hauser, T. U., Iannaccone, R., Stampfli, P., Drechsler, R., Brandeis, D., Walitza, S., \& Brem, S. (2014). The feedback-related negativity (FRN) revisited: New insights into the localization, meaning and network organization. NeuroImage, 84, 159-168. doi:https://doi. org/10.1016/j.neuroimage.2013.08.028

Holroyd, C. B., Krigolson, O. E., \& Lee, S. (2011). Reward positivity elicited by predictive cues. NeuroReport, 22(5), 249-252. doi: https://doi.org/10.1097/WNR.0b013e328345441d

Hu, X. M., Kim, A., Siwek, N., \& Wilder, D. (2017a). The Facebook paradox: Effects of Facebooking on individuals' social relationships and psychological well-being. Frontiers in Psychology, 8, 87. doi: https://doi.org/10.3389/fpsyg.2017.00087

Hu, X. M., Xu, Z. H., \& Mai, X. Q. (2017b). Social value orientation modulates the processing of outcome evaluation involving others. Social Cognitive and Affective Neuroscience, 12(11), 1730-1739. doi:https://doi.org/10.1093/scan/nsx102
Jung, T. P., Makeig, S., Humphries, C., Lee, T. W., McKeown, M. J., Iragui, V., \& Sejnowski, T. J. (2000). Removing electroencephalographic artifacts by blind source separation. Psychophysiology, 37(2), 163-178. doi:https://doi.org/10.1111/1469-8986.3720163

Kang, P., Lee, Y., Choi, I., \& Kim, H. (2013). Neural evidence for individual and cultural variability in the social comparison effect. Journal of Neuroscience, 33(41), 16200-16208. doi:https://doi. org/10.1523/jneurosci.5084-12.2013

Kang, S. K., Hirsh, J. B., \& Chasteen, A. L. (2010). Your mistakes are mine: Self-other overlap predicts neural response to observed errors. Journal of Experimental Social Psychology, 46(1), 229-232. doi: https://doi.org/10.1016/j.jesp.2009.09.012

Kutas, M., Mccarthy, G., \& Donchin, E. (1977). Augmenting mental chronometry: The P300 as a measure of stimulus evaluation time. Science, 197(4305), 792-795. doi:https://doi.org/10.1126/science. 887923

Leng, Y., \& Zhou, X. L. (2010). Modulation of the brain activity in outcome evaluation by interpersonal relationship: An ERP study. Neuropsychologia, 48(2), 448-455. doi:https://doi.org/10.1016/j. neuropsychologia.2009.10.002

Leng, Y., \& Zhou, X. L. (2014). Interpersonal relationship modulates brain responses to outcome evaluation when gambling for/against others: An electrophysiological analysis. Neuropsychologia, 63, 205-214. doi:https://doi.org/10.1016/j.neuropsychologia.2014.08. 033

Li, P., Shen, Y., Sui, X., Chen, C., Feng, T., Li, H., \& Holroyd, C. B. (2013). The neural basis of responsibility attribution in decisionmaking. PLOS ONE, 8(11). doi:https://doi.org/10.1371/journal. pone.0080389

Luo, Y., Eickhoff, S. B., Hetu, S., \& Feng, C. L. (2018). Social comparison in the brain: A coordinate-based meta-analysis of functional brain imaging studies on the downward and upward comparisons. Human Brain Mapping, 39(1), 440-458. doi:https://doi.org/10. $1002 / \mathrm{hbm} .23854$

Luo, Y., Feng, C. L., Wu, T. T., Broster, L. S., Cai, H. J., Gu, R. L., \& Luo, Y. J. (2015). Social comparison manifests in event-related potentials. Scientific Reports, 5. doi:https://doi.org/10.1038/srep12127

Ly, M., Haynes, M. R., Barter, J. W., Weinberger, D. R., \& Zink, C. F. (2011). Subjective socioeconomic status predicts human ventral striatal responses to social status information. Current Biology, 21(9), 794-797. doi:https://doi.org/10.1016/j.cub.2011.03.050

Ma, Q. G., Shen, Q. A., Xu, Q., Li, D. D., Shu, L. C., \& Weber, B. (2011). Empathic responses to others' gains and losses: An electrophysiological investigation. NeuroImage, 54(3), 2472-2480. doi:https:// doi.org/10.1016/j.neuroimage.2010.10.045

Marco-Pallares, J., Kramer, U. M., Strehl, S., Schroder, A., \& Munte, T. F. (2010). When decisions of others matter to me: An electrophysiological analysis. BMC Neuroscience, 11, 86. doi:https://doi.org/10. 1186/1471-2202-11-86

Miltner, W. H. R., Braun, C., \& Coles, M. G. H. (1997). Event-related brain potentials following incorrect feedback in a time-estimation task: Evidence for a "generic" neural system for error detection. Journal of Cognitive Neuroscience, 9(6), 788-798. doi:https://doi. org/10.1162/jocn.1997.9.6.788

Morris, M. W., \& Peng, K. (1994). Culture and cause: American and Chinese attributions for social and physical events. Journal of Personality and Social Psychology, 67(6), 949-971. doi:https:// doi.org/10.1037/0022-3514.67.6.949

Nieuwenhuis, S., Aston-Jones, G., \& Cohen, J. D. (2005). Decision making, the P3, and the locus coeruleus-norepinephrine system. Psychological Bulletin, 131(4), 510-532. doi:https://doi.org/10. 1037/0033-2909.131.4.510

Oliveira, F. T. P., McDonald, J. J., \& Goodman, D. (2007). Performance monitoring in the anterior cingulate is not all error related: Expectancy deviation and the representation of action-outcome 
associations. Journal of Cognitive Neuroscience, 19(12), 19942004. doi:https://doi.org/10.1162/jocn.2007.19.12.1994

Proudfit, G. H. (2015). The reward positivity: From basic research on reward to a biomarker for depression. Psychophysiology, 52(4), 449-459. doi:https://doi.org/10.1111/psyp.12370

Qi, Y. Y., Wu, H. Y., Raiha, S., \& Liu, X. (2018). Social value orientation modulates context-based social comparison preference in the outcome evaluation: An ERP study. Neuropsychologia, 112, 135-144. doi:https://doi.org/10.1016/j.neuropsychologia.2018.02.028

Qiu, J., Yu, C. Y., Li, H., Jou, J. W., Tu, S., Wang, T., ... Zhang, Q. L. (2010). The impact of social comparison on the neural substrates of reward processing: An event-related potential study. NeuroImage, 49(1), 956-962. doi:https://doi.org/10.1016/j.neuroimage.2009.08. 025

Schreuders, E., Klapwijk, E. T., Will, G. J., \& Guroglu, B. (2018). Friend versus foe: Neural correlates of prosocial decisions for liked and disliked peers. Cognitive Affective \& Behavioral Neuroscience, 18(1), 127-142. doi:https://doi.org/10.3758/s13415-017-0557-1

Shen, Q., Jin, J., \& Ma, Q. G. (2013). The sweet side of inequality: How advantageous status modulates empathic response to others' gains and losses. Behavioural Brain Research, 256, 609-617. doi:https:// doi.org/10.1016/j.bbr.2013.08.043

Sutton, S., Braren, M., Zubin, J., \& John, E. R. (1965). Evoked-potential correlates of stimulus uncertainty. Science, 150(3700), 1187-1188. doi:https://doi.org/10.1126/science.150.3700.1187

Tesser, A., Millar, M., \& Moore, J. (1988). Some affective consequences of social-comparison and reflection processes: The pain and pleasure of being close. Journal of Personality and Social Psychology, 54(1), 49-61. doi:https://doi.org/10.1037/0022-3514.54.1.49

Varnum, M. E. W., Shi, Z. H., Chen, A. T., Qiu, J., \& Han, S. H. (2014). When "your" reward is the same as "my" reward: Self-construal priming shifts neural responses to own vs. friends' rewards. NeuroImage, 87, 164-169. doi:https://doi.org/10.1016/j. neuroimage.2013.10.042

Vazire, S. (2016). Editorial. Social Psychological and Personality Science, 7(1), 3-7. doi:https://doi.org/10.1177/1948550615603955

Wang, Y. W., Kuhlman, D. M., Roberts, K., Yuan, B., Zhang, Z., Zhang, W., \& Simons, R. F. (2017). Social value orientation modulates the FRN and P300 in the chicken game. Biological Psychology, 127, 89-98. doi:https://doi.org/10.1016/j.biopsycho.2017.04.012
Wang, Y. W., Yuan, B., Roberts, K., Wang, Y., Lin, C. D., \& Simons, R. F. (2014). How friendly is a little friendly competition? Evidence of self-interest and empathy during outcome evaluation. International Journal of Psychophysiology, 91(3), 155-162. doi:https://doi.org/ 10.1016/j.ijpsycho.2013.10.009

Weinberg, A., Luhmann, C. C., Bress, J. N., \& Hajcak, G. (2012). Better late than never? The effect of feedback delay on ERP indices of reward processing. Cognitive, Affective, \& Behavioral Neuroscience, 12(4), 671-677. doi:https://doi.org/10.3758/s13415012-0104-z

Weinberg, A., Riesel, A., \& Proudfit, G. H. (2014). Show me the money: The impact of actual rewards and losses on the feedback negativity. Brain and Cognition, 87, 134-139. doi:https://doi.org/10.1016/j. bandc.2014.03.015

White, K., \& Lehman, D. R. (2005). Culture and social comparison seeking: The role of self-motives. Personality and Social Psychology Bulletin, 31(2), 232-242. doi:https://doi.org/10.1177/ 0146167204271326

Wu, Y., Zhang, D. X., Elieson, B., \& Zhou, X. L. (2012). Brain potentials in outcome evaluation: When social comparison takes effect. International Journal of Psychophysiology, 85(2), 145-152. doi: https://doi.org/10.1016/j.ijpsycho.2012.06.004

Yeung, N., \& Sanfey, A. G. (2004). Independent coding of reward magnitude and valence in the human brain. Journal of Neuroscience, 24(28), 6258-6264. https://doi.org/10.1523/JNEUROSCI.4537-03. 2004

Zhang, D., Gu, R., Wu, T., Broster, L. S., Luo, Y., Jiang, Y., \& Luo, Y. (2013). An electrophysiological index of changes in risk decisionmaking strategies. Neuropsychologia, 51(8), 1397-1407. doi: https://doi.org/10.1016/j.neuropsychologia.2013.04.014

Zhu, X. R., Wang, L. L., Yang, S. Y., Gu, R. L., Wu, H. Y., \& Luo, Y. J. (2016). The motivational hierarchy between the personal self and close others in the Chinese brain: An ERP study. Frontiers in Psychology, 7, 1467. doi:https://doi.org/10.3389/fpsyg.2016.01467

Publisher's note Springer Nature remains neutral with regard to jurisdictional claims in published maps and institutional affiliations. 